\title{
A Curriculum for Diagnostic Reasoning: JGIM's Exercises in Clinical Reasoning
}

\author{
Akshai Subramanian, $M D^{1,2}$, Denise M. Connor, $M D^{1,2}$, Gabrielle Berger, $M D^{3}$, \\ Juan N. Lessing, MD FACS ${ }^{4}$, Neil Mehta, MBBS MS ${ }^{5}$, Reza Manesh, MD 6 , and Jeffrey Kohlwes, MD \\ $M P H^{1,2}$
}

'University of California, San Francisco, San Francisco, CA, USA; ${ }^{2}$ Veterans Affairs Medical Center, San Francisco, San Francisco, CA, USA; ${ }^{3}$ University of Washington, Seattle, WA, USA; ${ }^{4}$ University of Colorado School of Medicine, Aurora, CO, USA; ${ }^{5}$ Cleveland Clinic Lerner College of Medicine of

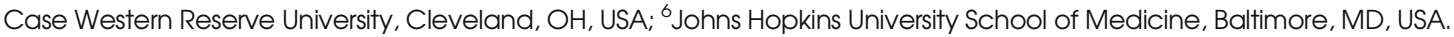

$\mathrm{J}$ Gen Intern Med 34(3):344-5 DOI: $10.1007 / \mathrm{s} 11606-018-4689-y$

(C) Society ofGeneral Internal Medicine (This is a U.S. government work and not under copyright protection in the U.S.; foreign copyright protection may apply) 2018

\section{INTRODUCTION}

In 2013, an estimated 251,000 deaths occurred due to medical error in the USA. ${ }^{1}$ Diagnostic errors make up a subset of these errors. Improving Diagnosis in Health Care advocates for training in clinical reasoning (CR) as a preventative strategy. ${ }^{2}$ However, there are few available resources to aid clinician-educators in teaching $\mathrm{CR}$.

To advance professional development in teaching foundational CR concepts, the Journal of General Internal Medicine (JGIM) initiated the Exercises in Clinical Reasoning (ECR) series. ${ }^{3}$ This series utilizes expert clinicians' discussions of challenging cases to demystify the CR process. We created a novel open-access online $\mathrm{CR}$ toolbox with downloadable teaching modules based on the print ECR series. ${ }^{4}$ By using this toolbox, teachers can engage learners in the case-based application of core CR concepts. In this report, we describe the process of developing this resource, its current usage statistics, and goals for future assessment and development.

\section{METHODS}

In 2015, five clinician-educators with CR expertise designed online educational tools to supplement the print version of the ECR series. The team reviewed the existing CR literature and identified four core CR concepts to highlight the following: Problem Representation, Illness Scripts, Dual Process Theory, and Diagnostic Schema. The panel then reviewed all 23 ECR publications, coded them by CR concept, and voted on the cases that best exemplified these four concepts. These manuscripts became the core content for the online curriculum. The panel drew on concepts of curriculum integration, which aim to teach learners in a comprehensive manner. ${ }^{5}$ Each teaching module was designed to include an introduction to the $\mathrm{CR}$ concept with a clinical example, access to the related ECR case, a PowerPoint

Published online October 17, 2018 teaching slide set, and a supplemental teacher's guide with suggested prompts and key discussion points. These modules were produced in teams, shared across groups for informal peer review, then posted to JGIM's website along with an introduction to the online toolbox. Module posts were accompanied by announcements from the Society of General Internal Medicine's (SGIM) Twitter account. We used Google Analytics software to collect information on site usage - including webpage views over time and source of entry to the ECR website.

\section{RESULTS}

The first online ECR modules on Illness Scripts, Problem Representation, and Dual Process Theory were posted in April 2016. Since then, there has been a steady rise in visitors to the ECR website, including several periods of significant traffic increase (Fig. 1). Seventy-nine percent of site visitors accessed the ECR website from organic web searches (i.e., without suggested results from sponsors such as SGIM), compared to $12 \%$ from direct links (e.g., via email), $8 \%$ from referrals from other webpages (such as JGIM web), and $1 \%$ from social media (Fig. 2).

\section{DISCUSSION}

We found continued growth in website visits 2 years after launching an online CR teaching toolbox. The majority of users accessed the site via organic web searches rather than through SGIM-specific links or social media posts. This data suggests high interest in and need for this open-access online curriculum.

Several next steps will help elucidate the practical impact of this resource. A better understanding of user demographics (e.g., experience level of teachers and learners), frequency and methods of use, barriers to use, and learners' impressions will guide further development of this online toolbox. We plan to include a brief online survey when users download materials to collect these data. We also plan to include space for qualitative comments, which will offer feedback on the successes, barriers, and challenges with using our modules. Ultimately, we hope to evaluate the impact of these materials on learners. As we study the needs of toolbox users, we plan to refine the 


\section{Pageviews}

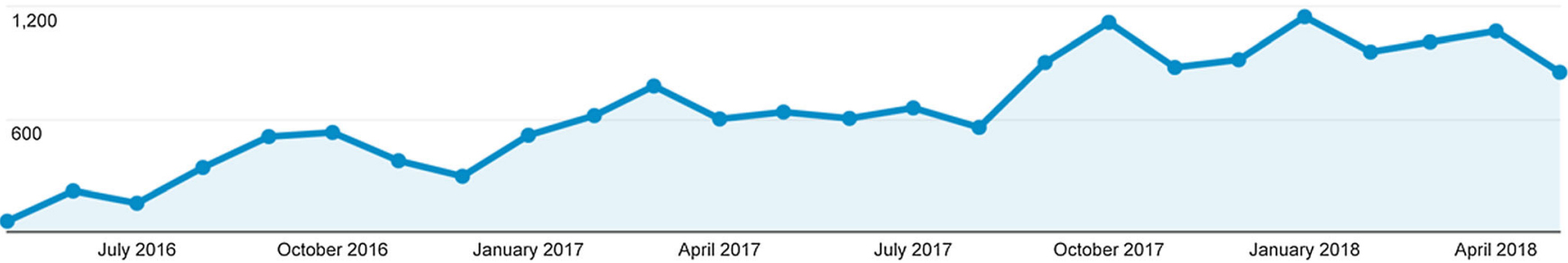

Figure 1 Exercises in Clinical Reasoning (ECR) webpage views per month (April 2016-May 2018).

existing modules. Future additions of ECR cases will highlight the existing core CR concepts and expand the breadth of the toolbox to cover additional CR concepts.

In this report, we describe the process of developing and implementing a novel open-access online toolbox to facilitate case-based training in CR. With further study, we hope to strengthen our online curriculum, with the goal of enhancing future clinicians' approaches to patient care through a better understanding of core CR concepts.

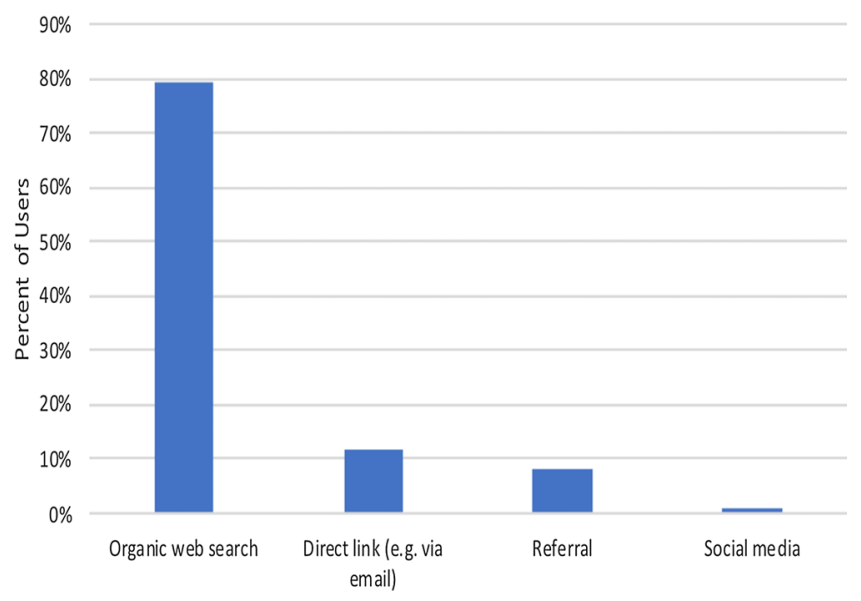

Figure 2 Route of Access to ECR Website. This figure summarizes how ECR users accessed the website. "Organic web search" represents access via search engine (e.g., Google Web Search).

"Direct link" represents access via a link to the ECR website (e.g., link shared by email). "Referral" links come from other ECRassociated webpages (e.g., JGIM web). "Social media" links to the website were predominantly shared on Twitter or Facebook.
Corresponding Author: Jeffrey Kohlwes, MD MPH; University of California, San Francisco, San Francisco, CA, USA (e-mail: Jeff. kohlwes@ucsf.edu).

\section{Compliance with Ethical Standards:}

Conflict of Interest: Dr. Manesh is supported by the Jeremiah A. Barondess Fellowship in the Clinical Transaction of the New York Academy of Medicine, in collaboration with the Accreditation Council for Graduate Medical Education (ACGME). No other conflict of interest exists, financial or others, to the best of our knowledge.

\section{REFERENCES}

1. Makary MA, Michael D. Medical error - the third leading cause of death in the US. BMJ. 2016;353:i2139.

2. National Academies of Sciences, Engineering, and Medicine. Improving Diagnosis in Health Care. Washington, DC: The National Academies Press; 2015.

3. Henderson M, Keenan C, Kohlwes J, Dhaliwal G. Introducing exercises in clinical reasoning. J Gen Intern Med. 2010;25(1):9.

4. Clinical Reasoning Exercises: Introduction to Exercise in Clinical Reasoning. Available at: https://www.sgim.org/web-only/clinical-reasoning-exercises. Accessed 9 June 2018.

5. Vergel J, Stentoft D, Montoya J. Extending the theoretical framework for curriculum integration in pre-clinical medical education. Perspectives on Medical Education. 2017;6(4):246-255. 